\title{
Feature Extraction Using Clustering of Protein
}

\author{
Isis Bonet $^{1}$, Yvan Saeys ${ }^{3}$, Ricardo Grau Ábalo ${ }^{1}$, María M. García ${ }^{1}$, \\ Robersy Sanchez ${ }^{2}$, and Yves Van de Peer ${ }^{3}$ \\ ${ }^{1}$ Center of Studies on Informatics, Central University of Las Villas, \\ Santa Clara, Villa Clara, Cuba \\ ${ }^{2}$ Research Institute of Tropical Roots, Tuber Crops and Banana (INIVIT), Biotechnology \\ Group, Santo Domingo, Villa Clara, Cuba \\ ${ }^{3}$ Department of Plant Systems Biology, Flanders Interuniversity Institute for Biotechnology \\ (VIB), Ghent University, Belgium \\ isisb@uclv.edu.cu, yvsae@psb.ugent.be, \\ \{rgrau, mmgarcia, robersy\}@uclv.edu.cu, \\ yves.vandepeer@psb.ugent.be
}

\begin{abstract}
In this paper we investigate the usage of a clustering algorithm as a feature extraction technique to find new features to represent the protein sequence. In particular, our work focuses on the prediction of HIV protease resistance to drugs. We use a biologically motivated similarity function based on the contact energy of the amino acid and the position in the sequence. The performance measure was computed taking into account the clustering reliability and the classification validity. An SVM using 10-fold crossvalidation and the $k$-means algorithm were used for classification and clustering respectively. The best results were obtained by reducing an initial set of 99 features to a lower dimensional feature set of 36-66 features.
\end{abstract}

Keywords: HIV resistance, SVM, clustering, k-means, similarity function.

\section{Introduction}

Nowadays, Bioinformatics is an area of research that is rapidly gaining in importance worldwide. It comprises several scientific fields, and is becoming a multidisciplinary science where input from e.g. machine learning research plays an important role.

Most of the problems in bioinformatics concern the analysis of DNA or protein sequences. In order to analyze these sequences, a common approach is to use the representation based on the sequence. However, in the case of protein sequences, more information could be conveyed by the three-dimensional structure. Studies on $3 \mathrm{D}$ structure are just another investigation problem in bioinformatics, yet unfortunately predicting the structure from the sequence is not evident. For this reason, we focus in this paper on a classification problem, starting from the primary sequence. The classification problem we consider here is the prediction of HIV drug resistance.

Given the protein sequence of protease, which is an important viral protein, the goal is to predict its resistance to drugs. The protease protein mutates constantly, and therefore resistance emerges easily after the application of a drug. 
To solve the resistance prediction problem, several machine learning techniques have been applied previously, such as decision trees, k-nearest neighbor technique (KNN) classifiers, and support vector machines (SVM) [1], [4], [10].

Usually, in problems on sequence analysis, the primary sequence is described using a feature for each amino acid. This representation consists of too much features. Given a problem where the length of the sequences is $n$ and taking into account that there exist 20 amino acids, this results in $20^{\mathrm{n}}$ possible features, the value of which correspond to the possible mutations of the sequence. The large number of possible mutations results in the fact that existing databases only represent a few of them, mostly having no known instances for each possible type of mutation. However, it can be considered that positions in the protein sequence that never mutate, will not influence the solution found by a machine learning algorithm.

On other hand, sequences are frequently very large, resulting in many possible features. These two observations motivate our work on reducing the dimensionality of the features that represent the protein sequence. Thinking in a first step, we start from a clustering process that looks for associations between amino acids in the sequence. Subsequently, an SVM is used as classification algorithm, where the idea is to find a small number of features that are sufficient to obtain good performance (i.e. values better or at less equivalent to the results obtained using all features).

\section{Methods}

\subsection{Datasets}

We used the information in the Stanford HIV Resistance Database Protease to develop our work because it is the one mostly used in the literature and it contains information about the genotype and phenotype for seven of the most widely used protease inhibitors. These inhibitors are: amprenavir (APV), atazanavir (ATV), nelfinavir (NFV), ritonavir (RTV), saquinavir (SQV), lopinavir (LPV) and indinavir (IDV). The genotype is described by the mutated positions with the corresponding change in amino acid. The phenotype is described by the resistance-fold based on the concentration for the drug to inhibit the viral protease as the resistance value. This database is available at http://hivdb.stanford.edu/cgibin/PIResiNote.cgi.

To eliminate missing values, the instances in the dataset with unknown changes in amino acid were discarded. Finally, seven databases were constructed, one for each drug. We took as reference sequence (wild type) the HXB2 protease and built the mutated sequences by changing the amino acid in the corresponding reported mutated positions. For the resistance-fold we used the 3.5 cut-off value, as previously reported in the literature for these drugs [2], [10]. If the drug resistance is greater than this cutoff value, the mutant is classified as resistant and otherwise as susceptible. In this way we obtain a binary (i.e. two-class) classification problem: $\{$ resistant, susceptible $\}$.

\subsection{Pattern Representation}

As in any classification problem, one of the first steps is looking for appropriate features to represent the input information. In problems of sequences analysis like 
this, it is common to use each amino acid as the information for the features. In this way, we will have the same number of features as the sequence has amino acids.

In some approaches, the simple representation of each sequence position being a binary vector of 20 elements (i.e. the amino acids) has been used. In that case, a value of 1 is given to the analyzed amino acid position and a 0 to all the others. Mutual information profiles have also been used to represent each sequence of the Protease enzyme [1].

Some methods using information of protein structure have also been reported in the literature [4]. The idea to use the information related to the 3D structure would be ideal, because this structure would allow us to know whether the drug has perfect contact with the protein to inhibit its functionality or not. However, the amount of available 3D data on HIV mutants is not enough in order to build a good training database. Since the amount of primary structure data is significantly higher than the number of 3D structures available, we used data based on primary structures. However, we use a representation of the sequence that allows to some extent to take into account some features related to the 3D-structure. In particular, we chose the amino acid contact energy as an adequate representation because it determines the (un)folding of the protein. Miyazawa and Jernigan (1994) showed that the contact energy changes the protein structure, and the substitution of a simple amino acid suffices to observe this [13], [14]. For this reason, the energy associated with each amino acid is used to represent the Protease sequence and will be referred to from now on as Energy (equation 1).

$$
\text { Energy: } A \rightarrow R
$$

where $\mathrm{A}$ is the set of 20 amino acids and $\mathrm{R}$ is the set of real numbers.

In particular, we have 7 databases, one for each drug. The problem was transformed into seven similar binary classification problems. The target function of each problem is the same (equation 2).

$$
\mathrm{F}: \mathrm{A} \rightarrow \mathrm{O}
$$

where $\mathrm{O}=$ \{resistant, susceptible $\}$ and $\mathrm{A} \subseteq \mathrm{R} 99$, because the database consists of sequences of the same length, namely 99 amino acids. Each element of A is a protease sequence identified by an amino acid vector. All amino acids are represented by their Energy, which is a real value.

As was explained in the introduction, the databases have some features that never change, which have the same value in all cases. One of the possible solutions to this problem is to find association between positions in the sequence and to do a transformation in the space building new features. Feature extraction can be a technique to compute new features taking into account the information of other features. 


\subsection{Feature Extraction}

In mathematical terms, dimensionality reduction is based on the following: given a $\mathrm{p}$ dimensional variable that defines the features $x=\left(x_{1} ; \ldots ; x_{p}\right)^{T}$; look for a lower dimensional representation of it $\left(s_{1} ; \ldots ; s_{k}\right)^{T}$ with $k<p$, that captures the content in the original data, according to some criterion.

Dimensionality reduction can be focused on feature selection or feature extraction. The first idea is based on finding a subset $s$ of features from the original data $x$. The second objective is focused on finding a transformation that can be applied to the space in all features.

Any of these techniques can be chosen. Features selection can help to select the positions with more influence in the resistance. However, in our case we did not want to lose the information of each position, as it could be biologically relevant in having an influence on the classification of the resistance. Therefore, we focus in this work on feature extraction.

Feature extraction consists of finding a matrix $\mathrm{C}_{\mathrm{k} \times \mathrm{p}}$, with $k<p$, from the matrix $\mathrm{X}_{\mathrm{n} \times p}$ where $n$ is the number of data in the database and $p$ the number of features. The idea is to transform the matrix $\mathrm{X}$ from the matrix $\mathrm{C}$ to obtain the matrix $\mathrm{U}$ representing the new features (equation 3 ).

$$
U^{T}=C \times X^{T}
$$

There are many feature extraction techniques commonly used in the literature for different problems [6]. Due to the characteristics of the problem, the transformation was focused on finding associations between the positions with biological meaning. In this way we can find groups of positions that relate to each other for reasons of resistance modeling. To compute the associations, there exist many statistical and artificial intelligence techniques. We select a clustering method to find the groups of positions in the sequence, as a fast way to do feature extraction. This technique has been used by other authors in other problems of pattern recognition [5], [15], [16].

Clustering is a technique that groups patterns into classes (termed clusters) based on similarity.

Any clustering task can be characterized by the following three principles [9]:

1. Pattern representation

2. Definition of a pattern similarity measure

3. Clustering algorithm

The first step was described in the section 2.2. Step 2 will be explained further in the paper. The last step includes the selection of the clustering algorithm In the literature there are several clustering techniques., reviewed by Jain, A. K. et al. (1999) [9]. One of them, $k$-means, is a classical algorithm and was chosen to be used in this work.

\subsubsection{K-Means}

$k$-means is a popular clustering algorithm which has been used in several applications. Given a set of $\mathrm{n}$ data points in $\mathrm{R}^{\mathrm{p}}$ and an integer $\mathrm{k}$, the $k$-means algorithm finds a set 
of $\mathrm{k}$ points $\mathrm{R}^{\mathrm{p}}$, called "centroids", and associates each point in the data to the nearest centroid. It is very important to select an appropriated similarity function [11].

This method has the disadvantage that it needs the number of clusters as input information (prior information). However, for our application this is not problematic, as we want to test all possible clusters number.

For the implementation of the clustering, we used Weka, which is a free software package that has implemented this algorithm. Furthermore, it has the advantage that it is easy to add a new similarity function. Weka is available at the following URL: http://www.cs.waikato.ac.nz/ml/weka.

\subsubsection{Similarity Function}

Selecting a similarity function is a key step for a good clustering process. There are already several functions defined in the literature, depending on the problem. Between the most popular are: Euclidean distance, Minkowski metric, squared Mahalanobis distance [3], [7].

In this paper, we defined our own similarity function, which has a biological meaning related to the clustering of sequence positions for drug resistance modeling With this type of function we can keep the interpretability of the data that precisely is a disadvantage of most feature extraction techniques.

As already discussed above, the data of our problem are defined by equations 1 and 2. Each element represents the Energy associated to the corresponding amino acid. However, we need to group the positions of the sequences taking into account the repercussions of the mutations in these positions. Using only the Energy information is not enough to build these groups. One can observe that the positions with the same amino acid will be member of the same group and this result is not valid. In order to keep the idea to find information relative to the 3D structure, we take the position as an additional type of information into account. We combined both Energy and position of each element in the sequence to obtain the following similarity function:

$$
d(k, l)=\sum_{i=1}^{n}\left(Z\left(k * e_{a_{k}}(i)\right)-Z\left(l * e_{a l}(i)\right)\right)^{2}
$$

where $\mathrm{Z}(\mathrm{x})$ represents the $\mathrm{Z}$-score normalization of the variable $\mathrm{x}$, defined in the equation 5:

$$
z(X)=\frac{X-\bar{X}}{S_{x}}
$$

Here, $\bar{X}$ is the mean of $X$ and $S_{x}$ is the standard deviation. In our case:

You can define $k^{*} e_{a k}=X_{1}$ and $l^{*} e_{a l}=X_{2}$, and one can consider equation 4 as the square of the Euclidean distance (see equation 6). 


$$
d(k, l)=\sum_{i=1}^{n}\left(Z\left(X_{1}(i)\right)-Z\left(X_{2}(i)\right)\right)^{2}
$$

\subsection{Classification Method}

\subsubsection{SVM}

Support Vector Machines (SVM) are a technique developed by Vapnik in 1996 from statistical learning theory [17]. SVM have become an important machine learning technique for many pattern recognition problems, especially in computational biology. For SVM training and testing we also used the Weka software.

\subsubsection{Performance Measure}

The performance measures used in this work were divided in two parts: one to evaluate the clustering, and the other to evaluate the classification.

To evaluate the classification results we use the Receiver Operating Characteristic curve (ROC curve) to evaluate the results of an SVM [12].

ROC curves have the advantage that they encapsulate all information contained in the confusion matrix. It is a plot of the true positive rate (TPr) against the false positive rate (FPr). To measure the classification performance, we used the area under the curve (AUC) [8].

\section{Results and Discussion}

The objective of this paper is focused on searching the best features to predict HIV drug resistance starting from feature extraction. We used $k$-means as clustering algorithm to find associations between the amino acids that describe the protein sequence. In a next step, we used an SVM as a classification method, and compared the results obtained using all features to those using the new features derived from the clustering.

First, we train the SVM using all features and afterwards, we applied $k$-means for all possible values of $k, k=1, \ldots, p-1$ (p denotes the sequence length). With small values of $\mathrm{k}$ we obtained good results, as is shown in Table 1. The first row in this table represents the results for all features (i.e. 99 amino acids). The rows 2 and 3 describe the results of 25 and 28 clusters respectively for each drug. As can be observed, the results for 25 clusters are better or at least as good as those using all features, for 6 of the 7 drugs. Using 28 clusters we already obtain good results for all drugs.

Table 1. Prediction performance (AUC) of SVM for the seven drugs using 99, 25 and 28 features

\begin{tabular}{llllllll}
\hline $\begin{array}{l}\text { Number } \\
\text { of features }\end{array}$ & APV & ATV & IDV & NFV & RTV & SQV & LPV \\
\hline 99 & 83.5 & 75.2 & 89.3 & 85.7 & 89.9 & 85.2 & 65 \\
25 & $\mathbf{8 3 . 6}$ & $\mathbf{8 1}$ & $\mathbf{8 9 . 8}$ & 83.8 & $\mathbf{9 2 . 9}$ & $\mathbf{8 7 . 6}$ & $\mathbf{7 7 . 2}$ \\
28 & 84 & 80.2 & 90.3 & $\mathbf{8 5 . 9}$ & 92.7 & 86.8 & 78.1 \\
\hline
\end{tabular}


With these results, however, we only check the validity of the SVM prediction. In this case we use a non-supervised technique (k-means) and a supervised technique (SVM). To do the validation of the results we need to check the validity of the classification, but we also need to check the reliability of the clustering.

In short, our process is based on the following steps:

1. Run $k$-means with the matrix $\mathrm{X}^{\mathrm{T}}$ for $k=1,2, . ., p$ - 1 . Where $k$ is the number of clusters and $p$ is the number of features.

2. Check clustering reliability. Compute the subset SK of reliable clusters number.

3. Build new features and new data matrix.

4. Run SVM for each $s k_{i} \in \mathrm{SK}$.

5. Check validity of results.

In the first step, we use the algorithm k-means implemented in Weka, with a variation of the similarity function, as was shown in equation 4 . This algorithm is run with all possible values of $k$.

However, the way to check the performance of a clustering process depends on the distribution of the elements in the clusters. In other words, if an element is very far from the centroid of a cluster, this could means that we do not have chosen enough clusters to group our data, i.e. this element could define another cluster.

To check the reliability, we defined another measure that from now on will be called "maxim centroid distance" (MCD) defined as follow:

$$
M C D=\max \left(C D\left(f_{i}\right)\right)
$$

where $\mathrm{F}$ is the feature set for clustering. For each $f_{i} \in \mathrm{F}, C D\left(f_{i}\right)$ represent the distance of the feature $f_{i}$ to the centroid of the corresponding cluster.

A value of MCD being too large is not desirable, because it would mean that at least one of the clusters contains some points that are located too far from the centroid. In order to answer the question of finding an appropriate value for MCD, we first need to analyze the similarity function (defined in equation 4) into more detail. The normalization of a variable using equation 5 guarantees that the mean and the standard deviation will be 0 and 1 respectively. The difference of two variables, normalized in this way, has mean 0 and constant variance 2 . Based on a basic probability theory the square sum of variables with normal distribution, mean zero and constant variance has a chi-square $\left(\chi^{2}\right)$ distribution. For this reason, equation 4 is $\chi^{2}$ distributed.

Due to the $\chi^{2}$ distribution of the similarity function, we can do a reliability analysis

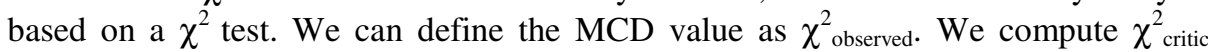
depending on the number " $n$ " of proteins and the level of significance $\alpha$. We work with $\alpha=0.05$ i.e., in such a way that the probability or $\chi^{2}$ was greater than $\chi_{\text {critic }}^{2}$ is lesser than 0.05 . This constant $\chi_{\text {critic }}^{2}$ will be compared with $\chi_{\text {observed }}^{2}$ (for each $k$ of clustering). That is, the idea is the following: if the maximum square Euclidean distance between the instances and the centroid $\left(\chi_{\text {observed }}^{2}\right)$ is greater than the value of 
$\chi_{\text {critic }}^{2}$ we have too few clusters. The objective is then to take a low number of clusters, without elements being too far from the corresponding centroids.

The second step is then to check the reliability of the clustering technique, in other words, to look for the minimum number of clusters needed to describe the variability of the data, and select the subset SK of value of $k$, which $\chi_{\text {observed }}^{2}<\chi_{\text {critic. }}^{2}$

The next step is based on the computation of the matrix $U$ (defined in equation 3 ), after finding the matrix $\mathrm{C}$ in the previous step with the clustering. For a given $k$, a clustering with $k$ clusters is obtained. In general, the elements $c_{i j}$ of the matrix $\mathrm{C}$ represent the membership of feature $j\left(f_{i}\right)$ to the cluster $i\left(\mathrm{~K}_{\mathrm{i}}\right)$ as shown the following equation:

$$
c_{i j}= \begin{cases}1 & \text { if } f_{j} \in K_{i} \\ 0 & \text { in other cases }\end{cases}
$$

SK is the subset of all clusters chosen in the previous step. For each $s k_{i} \in$ SK we build a new database. The database $i$ has $s k_{i}$ features computed from the matrix $\mathrm{U}$ defined in equation 3.

For each $s k_{i}$, a matrix $\mathrm{C}_{\mathrm{p} \times \mathrm{ki}}$ is represented in order to compute the matrix $\mathrm{U}^{\mathrm{T}}$, where $\mathrm{U}$ represents the new database with the new features. This database will have as features the clusters present in C. Each feature represents a cluster and it is computed as the sum of all features grouped in the cluster.

In short, in this step for each $s k_{i} \in \mathrm{K}$, we compute the matrix $\mathrm{U}$, which represents a new database. An SVM is then trained for this new database using Weka.

In the last step we check the classification performance of the SVM using AUC criterion, using 10 -fold crossvalidation.

Table 2. Classification measure (AUC ROC) of the better cluster for each database take into account the classification validity and clustering reliability

\begin{tabular}{llllllll}
\hline Clusters & APV & ATV & IDV & NFV & RTV & SQV & LPV \\
\hline $\mathbf{3 6}$ & & & & & 94 & & \\
$\mathbf{3 9}$ & & & & & & 82.3 \\
$\mathbf{4 1}$ & & & & & & \\
$\mathbf{5 4}$ & & 84.3 & & & & & \\
$\mathbf{6 2}$ & 85 & & & & & & \\
$\mathbf{6 5}$ & & & 92.2 & & & & \\
$\mathbf{6 6}$ & & & & 89.7 & & \\
\hline
\end{tabular}

Table 2 shows the results obtained in the process described above for the seven databases. It can be observed that the best results for each drug are obtained using a different number of clusters. These results could be expected, because the associations of positions are related in a different way with regard to each drug. In all cases we obtained results that were better than those obtained using all features. This demonstrates that the clustering process effectively reduces the dimensionality. 
Table 3. Comparison of the best cluster for each drug with previuos results. Prediction performance is measured in terms of accuracy. (1) Results by James (2004) (2) Results by Beerenwinkel et al. (2002). (3) Results using SVM with all features. (4) Results using SVM with the features computed from the best clustering.

\begin{tabular}{lllllll}
\hline Drug & $\begin{array}{l}\text { KNN } \\
(\mathbf{1})\end{array}$ & $\begin{array}{l}\text { Dtree } \\
(\mathbf{1})\end{array}$ & $\begin{array}{l}\text { New } \\
\text { DTree } \\
(\mathbf{1})\end{array}$ & $\begin{array}{l}\text { Dtree } \\
(\mathbf{2})\end{array}$ & $\begin{array}{l}\text { SVM } \\
(\mathbf{3})\end{array}$ & $\begin{array}{l}\text { SVM } \\
(\mathbf{4})\end{array}$ \\
\hline APV & 80.9 & 75.8 & 75.8 & 87.4 & 83.5 & 87.3 \\
ATV & & & & & 75.2 & $\mathbf{8 4 . 3}$ \\
IDV & 80.6 & 85 & 85.5 & 89.1 & 89.3 & $\mathbf{9 2}$ \\
NFV & 73.6 & 91.8 & 93.7 & 88.5 & 85.7 & 93.1 \\
RTV & 82 & 89 & 89.5 & 89.8 & 89.9 & $\mathbf{9 4 . 1}$ \\
SQV & 81.7 & 80 & 85.7 & 87.5 & 85.2 & $\mathbf{8 9 . 4}$ \\
LPV & 81.1 & - & 89.5 & - & 65 & $\mathbf{9 1 . 4}$ \\
\hline
\end{tabular}

Table 3 shows the comparison between the results obtained previously by James (2004) and Beerenwinkel (2002) [1],[10]. The column 5 represents the results using SMV with the 99 features and these are similar or better than the previous results obtained by James or Beerenwinkel. All results in this table are described using the accuracy as classification measure. Last column represent the results based on the best clustering described in the Table 2 for each drug. Only in APV and NFV the results are similar to the best results obtained previously, in the rest of the cases, our new method performs best.

\section{Conclusions}

In this paper, we focused on the problem of predicting Human Immunodeficiency Virus (HIV) Drug Resistance. We applied a clustering technique as a feature extraction method to sequence features in protein sequences. In applying this technique, a new similarity function with a biological meaning was defined, in order to look for associations between positions in the protein sequence of HIV protease.

We define a process to find the appropriate number of cluster based on the reliability of the results obtained with the k-means algorithm as a clustering method.

SVMs were then used to classify, and averaging using a 10-fold cross-validation we can conclude that the clustering process is a good way to do feature extraction, as well as reduce the dimensionality of the problem. We compared the results to those using all features, and to existing techniques for resistance prediction, concluding that our approach yields better results.

Acknowledgments. This work was developed in the framework of a collaboration program supported by VLIR (Vlaamse InterUniversitaire Raad, Flemish Interuniversity Council, Belgium). 


\section{References}

1. Beerenwinkel, N., Schmidt, B., Walter, H., Kaiser, R., Lengauer, T., Hoffmann, D., Korn, K., Selbig, J.: Diversity and complexity of HIV-1 drug resistance: A bioinformatics approach to predicting phenotype from genotype. PNAS 99 (2002) 8271-8276

2. Beerenwinkel, N., Daumer, M., Oette, M., Korn, K., Hoffmann, D., Kaiser, R., Lengauer, T., Selbig, J., Walter, H.: Geno2pheno: estimating phenotypic drug resistance from HIV-1 genotypes. Nucl. Acids Res. 31 (2003) 3850-3855

3. Bergo, A.: Text Categorization and Prototypes. www.illc.uva.nl/Publications/ ResearchReports/MoL-2001-08.text.pdf. (2001)

4. Cao, Z.W., Han, L.Y., Zheng, C.J., Ji, Z.L., Chen, X., Lin, H.H., Chen, Y.Z.: Computer prediction of drug resistance mutations in proteins. Drug Discovery Today 10 (2005) 521-529

5. Duda, R.O., Hart, P.E., Stork, D.G.: Pattern Classification. Wiley-Interscience (1997)

6. Fodor, I.K.: A survey of dimension reduction techniques. LLNL technical report. UCRLID-148494. (2002)

7. Gabrielsson, S.: MOVSOM-II- analysis and visualization of movieplot clusters. http://www.pcpinball.com/movsom. (2004)

8. Hanley, J.A., McNeil, B.J.: The meaning and use of the area under a receiver operating characteristic (ROC) curve. Radiology 143 (1982) 29-36

9. Jain, A.K., Murty, M.N., Flynn, P.J.: Data Clustering: A review. ACM Computing Surveys 31 (1999)

10. James, R.: Predicting Human Immunodeficiency Virus Type 1 Drug Resistance from Genotype Using Machine Learning. University of Edinburgh (2004)

11. McQueen, J.: Some methods for classification and analysis of multivariate observations. Fifth Berkeley Symposium on Mathematical Statistics and Probability (1967) 182-297

12. Metz, C.E.: Basic principles of ROC analysis. Seminars in Nuclear Medicine 8 (1978) 283-298

13. Miyazawa, S., Jernigan, R.L.: Protein stability for single substitution mutants and the extent of local compactness in the denatured state. Protein Eng. 7 (1994) 1209-1220

14. Miyazawa, S., Jernigan, R.L.: Residue Potentials with a Favorable Contact Pair Term and an Unfavorable High Packing Density Term, for Simulation and Threading. J. Mol. Biol. 256 (1996) 623-644

15. Slonim, N., Tishby, N.: The power of word clusters for text classification. 23rd European Colloquium on Information Retrieval Research (2001)

16. Steinbach, M., Karypis, G., Kumar, V.: A comparison of document clustering techniques. KDD Workshop on Text Mining (2000)

17. Vapnik, V.: The Nature of Statistical Learning Theory. Springer-Verlag, New York (1995) 